\title{
EXTENSION OF WIENER'S TAUBERIAN IDENTITY AND MULTIPLIERS ON THE MARCINKIEWICZ SPACE
}

BY

KA-SING LAU ${ }^{1}$

\begin{abstract}
This is a continuation of the work of Bertrandias, Lee and Lau on Wiener's generalized harmonic analysis. Among the other results, we extend Wiener's Tauberian identity to cover a larger class of functions; we characterize the multipliers on the Marcinkiewicz space $\Re^{2}$, and we obtain a Tauberian theorem on $\Re^{2}$ with full generality.
\end{abstract}

1. Introduction. For a complex valued Borel measurable function $f$ on $R$ such that $\lim _{T \rightarrow \infty}(2 T)^{-1} \int_{-T}^{T}|f(x)|^{2} d x$ exists, Wiener [13] defined the integrated Fourier transformation (Wiener transformation) $g=W(f)$ of $f$ as

$$
g(u)=\frac{1}{2 \pi}\left(\int_{-\infty}^{-1}+\int_{1}^{\infty} f(x) \frac{e^{-i u x}}{-i x} d x+\int_{-1}^{1} f(x) \frac{e^{-i u x}-1}{-i x} d x\right) .
$$

He then introduced a Tauberian theorem and proved that the mean square module of the above function $f$ equals the quadratic variation of its transformation $g$, i.e.

$$
\lim _{T \rightarrow \infty} \frac{1}{2 T} \int_{-T}^{T}|f(x)|^{2} d x=\lim _{\varepsilon \rightarrow 0^{+}} \frac{1}{2 \varepsilon} \int_{-\infty}^{\infty}|g(u+\varepsilon)-g(u-\varepsilon)|^{2} d u .
$$

The above transformation and identity were used by Wiener to study the spectra of certain important classes of functions which are not covered by classical harmonic analysis (cf. $[13,15,16])$.

For each $f \in L_{\text {loc }}^{2}(R)$, we let

$$
\|f\|=\|f\|_{\mathscr{R}^{2}}=\varlimsup_{T \rightarrow \infty}\left(\frac{1}{2 T} \int_{-T}^{T}|f(x)|^{2} d x\right)^{1 / 2}
$$

and $\mathfrak{R}^{2}=\left\{f: f \in L_{\text {loc }}^{2}(R),\|f\|<\infty\right\}$. We call $\Re^{2}$ the Marcinkiewicz space [10]. For any Borel measurable function $g$ on $R$, let

$$
\|g\|=\|g\|_{\mathcal{V}^{2}}=\varlimsup_{\varepsilon \rightarrow 0^{+}}\left(\frac{1}{2 \varepsilon} \int_{-\infty}^{\infty}|g(u+\varepsilon)-g(u-\varepsilon)|^{2} d u\right)^{1 / 2}
$$

and $\mathfrak{V}^{2}=\{g: g$ is Borel measurable and $\|g\|<\infty\}$. Such a class of functions was first investigated by Hardy and Littlewood [5] and is called the integrated Lipschitz class. By identifying functions whose differences have zero norm, both $\Re^{2}$ and $\mathfrak{V}^{2}$

Received by the editors January 26, 1982.

1980 Mathematics Subject Classification. Primary 42A45; Secondary 42A38.

${ }^{\prime}$ Supported by NSF Grant MCS7903638. Part of the work was done while the author was the Carl Beck Research Fellow to the Sophia University, Bulgaria. 
are Banach spaces [9]. Note that the identity (1.2) implies that $W$ is an isometry on the nonlinear subspace

$$
\mathscr{W}^{2}=\left\{f \in \mathfrak{N}^{2}: \lim _{T \rightarrow \infty} \frac{1}{2 T} \int_{-T}^{T}|f(x)|^{2} d x \text { exists }\right\} .
$$

In [9] Lee and the author have proved that the Wiener transformation $W$ is actually an isomorphism from $\Re^{2}$ onto $\widetilde{V}^{2}$ and the exact isomorphic constants are found. In [8], the author has investigated the class of convolution operators on $\Re^{2}$, and on $\mathcal{V}^{2}$ under the Wiener transformation. It is the main purpose of the present paper to carry on this study. Among the other results we extend Wiener's Tauberian identity (1.2) to a larger class of functions; we obtain different characterizations of multipliers on $\mathfrak{R}^{2}$, and we give a Tauberian theorem on $\mathfrak{R}^{2}$ with full generality.

Our paper is organized as follows.

In $\S 2$, we present some pertinent results, and use the Wiener transformation to obtain a characterization of the closed subspace $\Re_{c}^{2}$ of functions $f$ in $\Re^{2}$ which are continuous under translation, i.e. $\left\|\tau_{h} f-f\right\| \rightarrow 0$ as $h \rightarrow 0$. The space $\mathfrak{N}_{c}^{2}$ is the most important subspace in $\mathfrak{T}^{2}$ in our consideration.

In $§ 3$, the main result is Lemma 3.3. By using this, we offer an alternative proof of Wiener's Tauberian identity (1.2) and extend the identity to larger classes of functions (Theorem 3.4, Theorem 3.5).

In $\S 4$, we use Lemma 3.3 to study the covariance functions and spectral measures of $f$ in $\mathfrak{N}^{2}$.

The main theorems of this paper are in $\S 5$. For any operator $\Phi$ on $\mathfrak{K}^{2}$, the Wiener transformation defines a unique operator $\Phi^{\hat{\theta}}$ on $\mathfrak{V}^{2}$ which satisfies

$$
W(\Phi f)=\hat{\Phi}(W f), \quad f \in \Re^{2} .
$$

If $\Phi$ is a convolution operator defined by $\Phi(f)=\mu * f, f \in \mathfrak{N}^{2}$, where $\mu$ is a bounded regular Borel measure, then $\Phi^{\hat{S}}$ is given by $\hat{\Phi}(g)=\hat{\mu} \cdot g, g \in \mathcal{V}^{2}$, where $\hat{\mu}$ is the Fourier-Stieltjes transformation of $\mu$ [8]. Let $\left\langle\tau_{h}\right\rangle^{-}$be the strong operator closure of the subspace generated by the translation operators $\tau_{h} f, h \in R$. For $f \in \mathfrak{N}^{2}$, let $\left\langle\tau_{h} f\right\rangle^{-}$be the closed subspace generated by $\tau_{h} f, h \in R$. An operator $\Phi$ : $\mathfrak{N}_{c}^{2} \rightarrow \mathfrak{N}_{c}^{2}$ is called subordinative $[3,11]$ if $\Phi(f) \in\left\langle\tau_{h} f\right\rangle^{-}$for all $f \in \mathfrak{K}_{c}^{2}$. We prove the following theorem (Theorem 5.3).

THEOREM A. Let $\Phi$ be a bounded operator on $\Re_{c}^{2}$; then the following are equivalent:

(i) $\Phi$ is subordinative;

(ii) $\Phi \in\left\langle\tau_{h}\right\rangle^{-}$;

(iii) $\Phi$ belongs to the strong operator closure of the convolution operators;

(iv) there exists a unique $\phi \in C_{b}(R)$, the space of bounded continuous functions on $R$, such that $\hat{\Phi(}(g)=\phi \cdot g$ for all $g \in \mathcal{V}_{c}^{2}$.

We remark that Bertrandias [3] also showed that each subordinate operator $\Phi$ corresponds to a $\phi \in C_{b}(R)$ through some abstract representations. Our approach is quite different from his. We will call $\Phi$ a multiplier on $\mathfrak{N}_{c}^{2}$ if it satisfies either one of the above conditions. Also, we prove that (Theorem 5.2) 
THEOREM B. If we identify the space of multipliers on $\mathfrak{K}_{c}^{2}$ with $C_{b}(R)$, then the strong operator topology is stronger than the topology of uniform convergence on compact subsets of $R$. Moreover, the two topologies coincide on bounded subsets of $C_{b}(R)$.

Let $\Re_{r}^{2}$ denote the subspace of regular functions in $\Re^{2}$, i.e. $\lim _{T \rightarrow \pm \infty} \int_{T}^{T+a}|f|^{2}=0$ for all $a>0$, and let $\mathfrak{R}_{r c}^{2}=\mathfrak{M}_{r}^{2} \cap \mathfrak{N}_{c}^{2}$.

THEOREM C. Theorems $\mathrm{A}$ and $\mathrm{B}$ also hold for $\Re_{r c}^{2}$. In addition, if we assume that the restriction of $\Phi$ to $L^{2}(R)$ is a bounded linear operator on $L^{2}(R)$, then $\Phi$ is a multiplier on $\Re_{r c}^{2}$ if and only if $\Phi$ satisfies:

(i) $\Phi \tau_{h}=\tau_{h} \Phi \forall h \in R$;

(ii) $\lim _{T \rightarrow \infty} \frac{1}{2 T} \int_{-\infty}^{\infty}\left|\chi_{T} \Phi(f)-\Phi\left(\chi_{T} f\right)\right|^{2}=0$

where $\chi_{T}$ is the characteristic function on $[-T, T]$.

The conclusion on the subspace $\mathfrak{N}_{r}^{2}$ is different from $\mathfrak{N}_{c}^{2}$ and $\mathfrak{N}_{r c}^{2}$. The following result is a special case of Theorem 5.8, Corollary 5.9: Let $\hat{M}$ denote the set of $\hat{\mu}$ where $\mu$ is a bounded regular Borel measure on $R$. Consider $\hat{\mu}$ as an operator on $\mathcal{V}_{r}^{2}$ $\left(=W\left(\Re_{r}^{2}\right)\right)$; then the strong operator topology, the operator norm topology, and the uniform topology (as a subspace of $C_{b}(R)$ ) on $\hat{M}$ coincide.

As a direct application of the multiplier theorems, we obtain the following Tauberian theorem in $\S 6$. This generalizes a result of Wiener [16, Theorem 29; 8, Theorem 4.3].

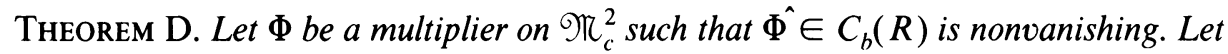
$f \in \mathfrak{N}_{c}^{2}$ satisfy

$$
\lim _{T \rightarrow \infty} \frac{1}{2 T} \int_{-T}^{T}|\Phi f|^{2}=0
$$

Then for any multiplier $\Psi$ on $\mathfrak{\Re}_{c}^{2}$,

$$
\lim _{T \rightarrow \infty} \frac{1}{2 T} \int_{-T}^{T}|\Psi f|^{2}=0 .
$$

The author wishes to thank Professor P. Masani and Dr. R. Nelson for bringing his attention to these problems in the seminar. Thanks also go to Professor I. Dimovski for some stimulating conversation while the author was visiting the Bulgarian Academy of Sciences.

2. Preliminaries. Let $\Re^{2}, \mathcal{V}^{2}$ and $W$ be defined as in $\S 1$. When there is no confusion, we will use the same notation $f \in \mathscr{T}^{2}\left(\mathscr{V}^{2}\right)$ to denote the function $f$ on $R$ as well as the equivalence class of functions in $\mathfrak{M}^{2}\left(\mathcal{V}^{2}\right.$, respectively). A function $f \in \mathfrak{M}^{2}$ is called regular if $\lim _{T \rightarrow \pm \infty} \frac{1}{2 T} \int_{T}^{T+1}|f(x)|^{2} d x=0$, or equivalently, $\lim _{T \rightarrow \pm \infty} \frac{1}{2 T} \int_{T}^{T+a}|f(x)|^{2} d x=0$ for all $a>0$. We will use $\mathfrak{M}_{r}^{2}$ to denote the closed subspace of all regular functions. Let $\tau_{h}, h \in R$, denote the translation operator defined by $\tau_{h} f(x)=f(x+h)$. A function $f \in \mathfrak{T}^{2}$ is said to be continuous under translation if $\left\|\tau_{h} f-f\right\| \rightarrow 0$ as $h \rightarrow 0$. We will let $\Re_{c}^{2}$ denote the class of functions and let $\mathfrak{M}_{r c}^{2}=\mathfrak{M}_{r}^{2} \cap \mathfrak{M}_{c}^{2}$. 
Let $M$ be the space of bounded regular Borel measures on $R$ and let $M_{1}$ denote the subspace of measures with finite first moment. In [8], we proved that each $\mu \in M_{1}$ defines a convolution operator $\Phi_{\mu}$ on $\Re^{2}$ by $\Phi_{\mu}(f)=\mu * f$ (pointwise). The definition can be extended to all $\mu \in M$ by taking limits under the strong operator topology.

Proposition 2.1. For each $\mu \in M$, the convolution operator $\Phi_{\mu}$ is a bounded linear operator on $\mathfrak{N}_{r}^{2}$ with $\left\|\Phi_{\mu}\right\|_{\mathfrak{R}_{r}^{2}}=\|\hat{\mu}\|_{\infty}$, where $\hat{\mu}$ is the Fourier transformation of $\mu$.

Let $\mathscr{D}^{1 / 2}$ denote the class of bounded functions $\phi$ on $R$ and satisfy $\phi(u+\varepsilon)-$ $\phi(u)=o\left(\varepsilon^{1 / 2}\right)$ uniformly on $u$. For each $g \in \mathcal{V}^{2}$, we can choose a $g^{\prime} \in \mathcal{V}^{2} \cap L^{2}$ such that $\left\|g-g^{\prime}\right\|_{\mho^{2}}=0\left[9\right.$, Theorem 3.3]. We define $\phi \cdot g$, for $\phi \in \mathcal{Q}^{1 / 2}$ and $g \in \mathcal{V}^{2}$, to be the equivalent class in $\mathcal{V}^{2}$ containing the pointwise multiplication $\phi \cdot g^{\prime}$. This multiplication is well defined [8] and

$$
\|\phi \cdot g\|=\varlimsup_{\varepsilon \rightarrow 0^{+}}\left(\frac{1}{2 \varepsilon} \int_{-\infty}^{\infty}|\phi(u)|^{2}|g(u+\varepsilon)-g(u-\varepsilon)|^{2} d u\right)^{1 / 2} .
$$

Also it was proved in [8, Theorem 3.6] that

Proposition 2.2. Let $W: \mathfrak{T}^{2} \rightarrow \mathfrak{V}^{2}$ be the Wiener transformation; then for any $\mu \in M$ such that $\hat{\mu} \in \mathscr{Q}^{1 / 2}$,

$$
W(\mu * f)=\hat{\mu} \cdot W(f) \quad \forall f \in \Re^{2} .
$$

It is easy to show that if $\mu \in M$ and the first moment exists, then $\hat{\mu} \in \mathscr{Q}^{1 / 2}$. In the following, we will make use of the above proposition to give a useful characterization of functions in $\pi_{c}^{2}$. This generalizes a result of Wiener [16, p. 160].

THEOREM 2.3. Let $f \in \mathfrak{R}^{2}$ and let $g=W(f)$. Then $f \in \mathfrak{K}_{c}^{2}$ if and only if

$$
\lim _{A \rightarrow \infty} \lim _{\varepsilon \rightarrow 0^{+}} \frac{1}{2 \varepsilon} \int_{-\infty}^{-A}+\int_{A}^{\infty}|g(u+\varepsilon)-g(u-\varepsilon)|^{2} d u=0 .
$$

Proof. Let $f \in \mathfrak{T}_{r}^{2}$; consider the sequence of functions $\left\{p_{n}\right\}_{n=1}^{\infty}$ where

$$
p_{n}(x)=\sqrt{\frac{2}{\pi}} \frac{n}{n^{2} x^{2}+1} .
$$

It is easy to show that $\left\|p_{n}\right\|_{1}=1$, the Fourier transformation of $p_{n}$ is $e^{-|u| / n}$ and belongs to $\mathscr{Q}^{1 / 2}$. We claim that $\left\|p_{n} * f-f\right\| \rightarrow 0$ as $n \rightarrow \infty$. Indeed, for any $0<\varepsilon<1$, there exists a $\delta>0$ such that for $0<|y|<\delta,\left\|\tau_{y} f-f\right\|<\varepsilon / 2$, and there exists an $n_{0}$ such that for $n>n_{0}$,

$$
\int_{R \backslash[-\delta, \delta]}\left|p_{n}(y)\right| d y<\frac{\varepsilon}{4\|f\|^{2}} .
$$

Hence for $n>n_{0}$,

$$
\begin{aligned}
\frac{1}{2 T} \int_{-T}^{T}\left|p_{n} * f(x)-f(x)\right|^{2} d x & \leqslant \frac{1}{2 T} \int_{-T}^{T}\left|\int_{-\infty}^{\infty}(f(x-y)-f(x)) p_{n}(y) d y\right|^{2} d x \\
& \leqslant \int_{-\delta}^{\delta}\left(\frac{1}{2 T} \int_{-T}^{T}|f(x-y)-f(x)|^{2} d x\right) p_{n}(y) d y+\frac{\varepsilon}{2} .
\end{aligned}
$$


By letting $T \rightarrow \infty$, we have $\left\|p_{n} * f-f\right\|^{2}<\varepsilon$. This completes the proof of the claim. Now by Proposition 2.2,

$$
\left(W\left(f-p_{n} * f\right)\right)(u)=\left(1-e^{-|u| / n}\right) g(u), \quad u \in R .
$$

Since $W$ is an isomorphism from $\Re^{2}$ onto $\widetilde{V}^{2}$,

$$
\lim _{n \rightarrow \infty} \varlimsup_{\varepsilon \rightarrow 0^{+}} \frac{1}{2 \varepsilon} \int_{-\infty}^{\infty}\left(1-e^{-|u| / n}\right)^{2}|g(u+\varepsilon)-g(u-\varepsilon)|^{2} d u=0 .
$$

That $\left|1-e^{-|u| / n}\right|>\frac{1}{2}$ for $|u|>n$ implies equality (2.2). To prove the sufficiency, we note that

$$
\left(W\left(f-\tau_{h} f\right)\right)(u)=\left(1-e^{i h u}\right) g(u), \quad u \in R .
$$

For $\varepsilon>0$, there exists an $A_{0}$ such that for $A>A_{0}$

$$
\varlimsup_{\varepsilon \rightarrow 0^{+}} \frac{1}{2 \varepsilon} \int_{-\infty}^{-A}+\int_{A}^{\infty}|g(u+\varepsilon)-g(u-\varepsilon)|^{2} d u<\frac{\varepsilon}{4} .
$$

Choose $\delta$ such that for $|h|<\delta,\left|1-e^{i h u}\right|<\varepsilon / 2\|g\|^{2}$ for $u \in\left[-A_{0}, A_{0}\right]$. Hence for $g \in \mathfrak{V}^{2}$

$$
\begin{aligned}
\left\|\left(1-e^{i h u}\right) g\right\|^{2} & =\varlimsup_{\varepsilon \rightarrow 0^{+}} \frac{1}{2 \varepsilon} \int_{-\infty}^{\infty}\left|1-e^{i h u}\right|^{2}|g(u+\varepsilon)-g(u-\varepsilon)|^{2} d u \quad(\text { by }(2.1)) \\
& \leqslant \varlimsup_{\varepsilon \rightarrow 0^{+}} \frac{1}{2 \varepsilon} \int_{[-A, A]}+\int_{R \backslash[-A, A]}\left|1-e^{i h u}\right|^{2}|g(u+\varepsilon)-g(u-\varepsilon)|^{2} d u \\
& \leqslant \frac{\varepsilon}{2}+\frac{\varepsilon}{2}=\varepsilon .
\end{aligned}
$$

It follows from the isomorphism of $W$ and equation (2.3) that $\left\|\tau_{h} f-f\right\| \rightarrow 0$ as $h \rightarrow 0$.

3. Some lemmas. In this section, we will develop some lemmas which lead to Theorems 3.4, 3.5 and Proposition 4.3.

Let $\Re$ denote the set of complex Borel measurable functions on $[0, \infty)$ such that $\sup _{T>0} \frac{1}{T} \int_{0}^{T}|f(x)| d x<\infty$. Let $\mathscr{B}_{1}$ denote the subset of all $f \in \mathscr{B}$ such that $\sup _{T>0} \frac{1}{T} \int_{0}^{T}|f(x)| d x \leqslant 1$. For any complex Borel measurable function $h$ on $R$, we will let $\tilde{h}(x)=\operatorname{ess~sup~}_{t \geqslant x}|h(t)|, x>0$. It is clear that $\tilde{h}$ is a decreasing function on $R$.

Lemma 3.1. Let $h$ be defined on $(0, \infty)$ such that $\tilde{h}$ is integrable on $(0, \infty)$. Then

(i) $\lim _{\alpha \rightarrow 0^{+}} \alpha h(\alpha)=0, \lim _{\beta \rightarrow \infty} \beta h(\beta)=0$;

(ii) $\lim _{\alpha \rightarrow 0^{+}} \int_{0}^{\alpha} f(T x) h(x) d x=0, \lim _{\beta \rightarrow \infty} \int_{\beta}^{\infty} f(T x) h(x) d x=0$ uniformly for all $f \in G_{1}$, and $T>0$.

Proof. (i) follows easily from the fact that $\tilde{h}$ is decreasing and integrable. To prove the first expression in (ii), we observe that for any $f \in \mathscr{B}_{1}, \alpha, T>0$, by changing a variable with $t=T x$ we have

$$
\left|\int_{0}^{\alpha} f(T x) d x\right|=\left|\alpha \cdot \frac{1}{\alpha T} \int_{0}^{\alpha T} f(t) d t\right| \leqslant \alpha .
$$


Let $\mu(a, x]=\tilde{h}(x+)-\tilde{h}(a+)$. Since $\tilde{h}$ is decreasing, $\mu$ is a negative measure. For any $T, \alpha>0$,

$$
\begin{aligned}
\left|\int_{0}^{\alpha} f(T x) h(x) d x\right| & \leqslant \int_{0}^{\alpha}|f(T x)| \tilde{h}(x) d x \\
& =\left(\int_{0}^{\alpha} f(T x) d x\right) \tilde{h}(\beta)-\int_{0}^{\alpha}\left(\int_{0}^{x} f(T x) d x\right) d \mu(x) \\
& \leqslant \alpha \tilde{h}(\alpha)-\int_{0}^{\alpha} x d \mu(x)=\int_{0}^{\alpha} \tilde{h}(x) d x .
\end{aligned}
$$

This completes the proof. The second identity is proved in [9, Proposition 4.2(ii)].

LEMMA 3.2. Let $h$ be as in Lemma 3.1. For any $\varepsilon>0$, let $0<\alpha<\beta$ be such that (i) $\alpha h(\alpha), \beta h(\beta)<\varepsilon$, (ii) $\left|\int_{0}^{\alpha} f^{\prime}(T x) h(x) d x\right|,\left|\int_{\beta}^{\infty} f^{\prime}(T x) h(x) d x\right|<\varepsilon$ for all $f^{\prime} \in \mathscr{G}_{1}$, $T>0$. Let $f \in \mathscr{B}_{1}, 0<a<b$ and $0 \leqslant c \leqslant 1$ satisfy

$$
\left|\frac{1}{T} \int_{0}^{T} f(x) d x-c\right|<\varepsilon \quad \forall T \in[a, b] .
$$

Then

$$
\left|\int_{0}^{\infty} f(T x) h(x) d x-c \int_{0}^{\infty} h(x) d x\right| \leqslant\left(8+\|h\|_{1}\right) \varepsilon \quad \forall T \in\left[\frac{a}{\alpha}, \frac{b}{\beta}\right] .
$$

Proof. Without loss of generality, we assume that $h$ has finite variation on any bounded intervals. Let $\mu_{h}(a, x]=h(x+)-h(a+)$. Then integration by parts and (3.1) yield

$$
\begin{aligned}
\int_{\alpha}^{\beta} f(T x) h(x) d x= & \left(\beta \cdot \frac{1}{\beta T} \int_{0}^{\beta T} f(x) d x\right) h(\beta)-\left(\alpha \cdot \frac{1}{\alpha T} \int_{0}^{\alpha T} f(x) d x\right) h(\alpha) \\
& -\int_{\alpha}^{\beta} x\left(\frac{1}{x T} \int_{0}^{x T} f(t) d t\right) d \mu_{h}(x)
\end{aligned}
$$

Hence for any $T \in[a / \alpha, b / \beta]$

$$
\begin{gathered}
\left|\int_{0}^{\infty} f(T x) h(x) d x-c \int_{0}^{\infty} h(x) d x\right| \leqslant\left|\int_{\alpha}^{\beta} f(T x) h(x) d x-c \int_{\alpha}^{\beta} h(x) d x\right|+4 \varepsilon \\
\leqslant c\left|\left(\beta h(\beta)-\alpha h(\alpha)-\int_{\alpha}^{\beta} x d \mu_{h}(x)\right)-\int_{\alpha}^{\beta} h(x) d x\right| \\
+\varepsilon\left(\beta|h(\beta)|+\alpha|h(\alpha)|+\left|\int_{\alpha}^{\beta} x d \mu_{h}(x)\right|+4 \varepsilon\right. \\
\quad(\text { by (3.2) and by the hypothesis on } c) \\
\leqslant \varepsilon\left(8+\|h\|_{1}\right) .
\end{gathered}
$$

The following is our main lemma. 
Lemma 3.3. Let $h$ be as in Lemma 3.1 and let $f \in \Re_{1}$. Suppose there exists $c>0$ and two sequences $\left\{T_{n}\right\}$ and $\left\{A_{n}\right\}$ such that:

(i) $\lim _{n \rightarrow \infty} T_{n}=\infty$ and $\lim _{n \rightarrow \infty}\left(A_{n} / T_{n}\right)=\infty$;

(ii) $\lim _{n \rightarrow \infty}\left(1 / S_{n}\right) \int_{0}^{S_{n}} f(x) d x=c$ for all $S_{n} \in\left[T_{n}, T_{n}+A_{n}\right]$.

Then there exists a sequence $\left\{T_{n}^{\prime}\right\}$ with $\lim _{n \rightarrow \infty} T_{n}^{\prime}=\infty$ and

$$
\lim _{n \rightarrow \infty} \int_{0}^{\infty} f\left(T_{n}^{\prime} x\right) h(x) d x=c \int_{0}^{\infty} h(x) d x .
$$

Proof. For any $\varepsilon>0$, let $\varepsilon_{1}=\varepsilon /\left(8+\|h\|_{1}\right)$. Let $\alpha, \beta$ be the corresponding number for $\varepsilon_{1}$ as in Lemma 3.2. Let $n_{0}$ be such that for $n \geqslant n_{0}$,

$$
\left|\frac{1}{T} \int_{0}^{T} f(x) d x-c\right|<\varepsilon_{1} \quad \forall T \in\left[T_{n}, T_{n}+A_{n}\right]
$$

and

$$
\frac{T_{n}+A_{n}}{\beta}>\frac{T_{n}}{\alpha}>\varepsilon_{1}^{-1} \quad(\text { by }(\mathrm{i})) .
$$

By Lemma 3.2,

$$
\left|\int_{0}^{\infty} f(T x) h(x) d x-c \int_{0}^{\infty} h(x) d x\right|<\varepsilon \quad \forall T \in\left[\frac{T_{n}}{\alpha}, \frac{T_{n}+A_{n}}{\beta}\right], \quad n \geqslant n_{0} .
$$

In particular, if we let $\varepsilon=\frac{1}{m}$ and let $T_{m}^{\prime}$ be $T_{n_{0}} / \alpha$ (which depends on $m$ ), then $\lim _{m \rightarrow \infty} T_{m}^{\prime}=\infty$ and

$$
\lim _{m \rightarrow \infty} \int_{0}^{\infty} f\left(T_{m}^{\prime} x\right) h(x) d x=c \int_{0}^{\infty} h(x) d x
$$

As an immediate application of the above lemma, we will extend the Wiener identity to a larger class of functions. Let $\chi_{A}$ denote the characteristic function of a set $A$.

THEOREM 3.4. Let $f_{0} \in \mathcal{Q} \int^{2}$. Let $\left\{T_{n}\right\},\left\{A_{n}\right\}$ be as in Lemma 3.3 with $c=\left\|f_{0}\right\|_{\mathfrak{K}^{2}}$, and let $A=\cup_{n=1}^{\infty}\left[T_{n}, T_{n}+A_{n}\right]$. Then the function $f=f_{0} \cdot \chi_{A}$ satisfies $\|f\|_{\Re^{2}}=$ $\|\boldsymbol{W}(f)\|_{\mathfrak{v}^{2}}$.

We remark that such an $f$ is easy to obtain by taking $\left\{T_{n}\right\},\left\{A_{n}\right\}$ sufficiently large.

Proof. Let $g=W(f)$. Then

$$
\begin{aligned}
g(u+\varepsilon)-g(u-\varepsilon) & =\frac{1}{2 \pi} \int_{-\infty}^{\infty} f(x) \frac{e^{i \varepsilon x}-e^{-i \varepsilon x}}{i x} e^{-i u x} d x \\
& =\frac{1}{2 \pi} \int_{-\infty}^{\infty} f(x) \frac{2 \sin \varepsilon x}{x} e^{-i u x} d x
\end{aligned}
$$

Hence $\left(\tau_{\varepsilon} g-\tau_{-\varepsilon} g\right)$ is the Fourier transformation of $\sqrt{\frac{2}{\pi}} f(x) \sin \varepsilon x / x$. The Plancherel Theorem implies that

$$
\begin{aligned}
\frac{1}{2 \varepsilon} \int_{-\infty}^{\infty}|g(u+\varepsilon)-g(u-\varepsilon)|^{2} d u & =\frac{1}{\varepsilon} \int_{-\infty}^{\infty}|f(x)|^{2} \frac{\sin ^{2} \varepsilon x}{\pi x^{2}} d x \\
& =\int_{-\infty}^{\infty}\left|f\left(\frac{x}{\varepsilon}\right)\right|^{2} \frac{\sin ^{2} x}{\pi x^{2}} d x
\end{aligned}
$$


By taking $F(x)=\left(|f(x)|^{2}+|f(-x)|^{2}\right) / 2$ and $h(x)=2 \sin ^{2} x / \pi x^{2}$,

$$
\|W f\|_{\mathfrak{r}^{2}}=\varlimsup_{T \rightarrow \infty} \int_{0}^{\infty} F(T x) h(x) d x .
$$

Similarly, we can define $F_{0}$ for $f_{0}$. Lemma 3.3 implies that

$$
\|f\|_{\mathscr{R}^{2}}=\lim _{n \rightarrow \infty} \int_{0}^{\infty} F\left(T_{n}^{\prime} x\right) h(x) d x \leqslant\|W f\|_{\mathscr{V}^{2}}
$$

for some sequence $\left\{T_{n}^{\prime}\right\}$ diverging to $\infty$. On the other hand, since $f \in \mathscr{W} \int^{2}$

$$
\begin{aligned}
\|W f\|_{\mathfrak{V}^{2}} & =\varlimsup_{T \rightarrow \infty} \int_{0}^{\infty} F(T x) h(x) d x \leqslant \varlimsup_{T \rightarrow \infty} \int_{0}^{\infty} F_{0}(T x) h(x) d x \\
& =\left\|W\left(f_{0}\right)\right\|_{\mathfrak{V}^{2}}=\left\|f_{0}\right\|_{\mathscr{N}^{2}}=\|f\|_{\mathfrak{R}^{2}} .
\end{aligned}
$$

A function $f \in \mathscr{T}^{2}$ is said to have slow oscillating quadratic mean if for any $\varepsilon>0$, there exists a positive function $\phi_{\varepsilon}$ on $[0, \infty)$ such that:

(i) $\lim _{T \rightarrow \infty}\left(\phi_{\varepsilon}(T) / T\right)=\infty$;

(ii) there exists $T_{\varepsilon}$ such that for $T>T_{\varepsilon}$

$$
\left.\left|\frac{1}{2 T} \int_{-T}^{T}\right| f(x)\right|^{2} d x-\frac{1}{2 T^{\prime}} \int_{-T^{\prime}}^{T^{\prime}}|f(x)|^{2} d x \mid<\varepsilon \quad \forall T^{\prime} \in[T, T+\phi(T)] .
$$

It is clear that if $f \in \mathcal{Q} \int^{2}$, then $f$ has slow oscillating quadratic mean by taking $\phi_{\varepsilon}(T)=\varepsilon T^{2}$.

THEOREM 3.5. Suppose $f \in \mathfrak{N}^{2}$ and has slow oscillating quadratic mean; then $\|f\|_{\mathscr{R}^{2}}=\|W(f)\|_{\mathbb{V}^{2}}$.

Proof. By choosing $\left\{T_{n}\right\}$ such that

$$
\lim _{n \rightarrow \infty} \frac{1}{2 T_{n}} \int_{-T_{n}}^{T_{n}}|f|^{2}=\|f\|_{\Re^{2}}
$$

and by the same proof as the last theorem, we have $\|f\|_{\mathscr{R}^{2}} \leqslant\|W f\|_{\mathfrak{V}^{2}}$. To prove the reverse inequality, we let $\left\{T_{n}^{\prime}\right\}$ be a sequence diverging to $\infty$ and

$$
\lim _{n \rightarrow \infty} \int_{0}^{\infty} F\left(T_{n}^{\prime} x\right) h(x) d x=\|W f\|_{\mathfrak{V}^{2}}
$$

For each $\varepsilon=\frac{1}{k}$, there exist $\alpha_{k}, \beta_{k}$ satisfying the conditions (i) and (ii) in Lemma 3.2. Choose a subsequence $\left\{T_{n_{k}}^{\prime}\right\}$ of $\left\{T_{n}^{\prime}\right\}$ such that $T_{k}=\alpha_{k} T_{n_{k}}^{\prime} \rightarrow \infty$ as $k \rightarrow \infty$, and

$$
\lim _{k \rightarrow \infty} \frac{1}{T_{k}} \int_{0}^{T_{k}} F(x) d x
$$

exists. The sequence of intervals $\left[T_{k}, T_{k}+\varphi_{1 / k}\left(T_{k}\right)\right]$ satisfies the conditions in Lemma 3.3, and from the proof of Lemma 3.3, the sequence $\left\{T_{n_{k}}^{\prime}\right\}$ satisfies

$$
\lim _{k \rightarrow \infty} \int_{0}^{\infty} F\left(T_{n_{k}}^{\prime} x\right) h(x) d x=\lim _{k \rightarrow \infty} \frac{1}{T_{k}} \int_{0}^{T_{k}} F(x) d x .
$$

This implies that

$$
\|W(f)\|_{\mathfrak{V}^{2}}=\lim _{k \rightarrow \infty} \frac{1}{T_{k}} \int_{0}^{T_{k}} F(x) d x \leqslant\|f\|_{\mathscr{\Re}^{2}}
$$


Corollary 3.6 (WiEner). Suppose $f \in \mathcal{Q} \int^{2}$; then $\|f\|_{\mathscr{N}^{2}}=\|W f\|_{\mathfrak{V}^{2}}$.

4. The covariance functions and measures. Let $f \in \Re_{c}^{2}$ and let

$$
c_{T}(h)=\frac{1}{2 T} \int_{-T}^{T} f(x+h) \overline{f(x)} d x .
$$

Then $\left\{c_{T}\right\}_{T>0}$ is a family of uniformly bounded continuous, positive definite functions on $R$. Consider $\left\{c_{T}\right\}_{T>0}$ as a subset of the space of complex valued functions on $R$ with the topology of pointwise convergence, it is contained in the compact set $\{\psi:|\psi(h)| \leqslant k \forall h \in R\}$ for some $k>0$. Moreover, on $\left\{\overline{c_{T}}\right\}_{T>0}$, the topology of pointwise convergence and the topology of uniform convergence on compact sets of $R$ coincide $[4, \S 5]$. Let

$$
C_{f}=\bigcap_{k=1}^{\infty}\left\{\overline{c_{T}: T \geqslant k}\right\},
$$

i.e. $C_{f}$ is the set of limit points of $\left\{c_{T}\right\}_{T>0}$ as $T \rightarrow \infty$. Each $\phi \in C_{f}$ is positive definite and $\phi$ is continuous (since $f \in \mathfrak{N}_{c}^{2}$ ). For each $c \in C_{f}$ the Bochner Theorem yields a bounded regular Borel measure $\mu$ satisfying

$$
c(h)=\int_{R} e^{i h u} d \mu(u), \quad h \in R .
$$

Let $g \in \mathcal{V}^{2}$ and let $\mu_{\varepsilon}$ be a bounded regular Borel measure on $R$ defined by

$$
\mu_{\varepsilon}(E)=\frac{1}{2 \varepsilon} \int_{E}|g(u+\varepsilon)-g(u-\varepsilon)|^{2} d u,
$$

where $E$ is a bounded Borel subset in $R$. Let $D_{g}$ denote the weak* limit of the set $\left\{\mu_{\varepsilon}\right\}_{\varepsilon>0}$ as $\varepsilon \rightarrow 0$, i.e. $D_{g}=\bigcap_{\delta>0}\left\{\mu_{\varepsilon}: 0<\varepsilon<\delta\right\}$.

For $f \in \mathfrak{N}_{c}^{2}$ and $\lim _{T \rightarrow \infty} \frac{1}{2 T} \int_{-T}^{T} f(x+h) f(x) d x$ which exists for all $h \in R$, Wiener [13] proved that both $C_{f}$ and $D_{g}, g=W(f)$, are single points and they are related by the formula

$$
\lim _{T \rightarrow \infty} \frac{1}{2 T} \int_{-T}^{T} f(x+h) \overline{f(x)} d x=\lim _{\varepsilon \rightarrow 0} \frac{1}{2 \varepsilon} \int_{-\infty}^{\infty} e^{i h u}|g(u+\varepsilon)-g(u-\varepsilon)|^{2} d u,
$$

i.e., $c(h)=\int_{-\infty}^{\infty} e^{i h u} d \mu(u)$ where $\{\mu\}=D_{g}$.

THEOREM 4.1. Given any bounded regular Borel measure $\mu$ on $R$, there exists a $g \in \mathcal{V}^{2}$ such that $D_{g}=\{\mu\}$.

Proof. Let $c(h)=\int_{R} e^{i h u} d \mu(u)$. In [3], Bertrandias constructed a function $f \in$ $\psi^{2} \cap \Re_{c}^{2}$ such that

$$
c(h)=\lim _{T \rightarrow \infty} \frac{1}{2 T} \int_{-T}^{T} f(x+h) \overline{f(x)} d x \quad \forall h \in R .
$$

Let $g=W(f)$. It follows from (4.1) that $D_{g}$ consists of a unique measure and equals $\mu$. 
Lemma 4.2. Let $f \in \mathfrak{M}^{2}$ and let $g=W(f)$. Suppose $f$ satisfies the following conditions: for any compact subset $K$ in $R$, there exist two sequences $\left\{T_{n}\right\}$ and $\left\{S_{n}\right\}$ such that:

(i) $\lim _{n \rightarrow \infty} T_{n}=\infty, \lim _{n \rightarrow \infty}\left(S_{n} / T_{n}\right)=\infty$;

(ii) $\lim _{n \rightarrow \infty}\left(1 / 2 T_{n}^{\prime}\right) \int_{0}^{T_{n}^{\prime}} f(x+h) \overline{f(x)} d x$ exists and equals $c(h) \forall T_{n}^{\prime} \in\left[T_{n}, T_{n}+S_{n}\right]$, $h \in K$.

Then there exists a sequence $\left\{\varepsilon_{n}\right\}$ which converges to zero and

$$
\lim _{n \rightarrow \infty} \frac{1}{2 \varepsilon_{n}} \int_{-\infty}^{\infty} e^{i h u}\left|g\left(u+\varepsilon_{n}\right)-g\left(u-\varepsilon_{n}\right)\right|^{2} d u=c(h) \quad \forall h \in K .
$$

Proof. Note that $e^{i h u}(g(u+\varepsilon)-g(u-\varepsilon))$ and $(g(u+\varepsilon)-g(u-\varepsilon))$ are the Fourier transformations of the functions

$$
\sqrt{\frac{2}{\pi}} f(x+h) \frac{\sin \varepsilon x}{x} \text { and } \sqrt{\frac{2}{\pi}} f(x) \frac{\sin \varepsilon x}{x}
$$

respectively. Hence

$$
\frac{1}{2 \varepsilon} \int_{-\infty}^{\infty} e^{i u h}|g(u+\varepsilon)-g(u-\varepsilon)|^{2} d u=\int_{-\infty}^{\infty} f\left(\frac{x+h}{\varepsilon}\right) \overline{f\left(\frac{x}{\varepsilon}\right)} \frac{\sin ^{2} x}{\pi x^{2}} d x .
$$

We then apply Lemma 3.3 to $F(x)=\frac{1}{2}(f(x+h) \overline{f(x)}+f(-(x+h)) \overline{f(-x)})$ and $h(x)=2 \sin ^{2} x / \pi x^{2}$ to obtain the conclusion.

The following proposition will play an important role in the next section.

Proposition 4.3. Let $\left\{\mu_{n}\right\}$ be a sequence of positive regular Borel measures on $R$ with $\left\|\mu_{n}\right\| \leqslant 1$. Then there exists a $g \in \mathfrak{V}^{2}$ such that $D_{g}$ contains $\left\{\mu_{n}\right\}$.

Proof. For each $\mu_{n}$, there exists an $f_{n} \in \mathcal{O} \int^{2} \cap \Re_{c}^{2}$ such that

$$
\lim _{T \rightarrow \infty} \frac{1}{2 T} \int_{-T}^{T} f_{n}(x+h) \overline{f_{n}(x)} d x=\int_{-\infty}^{\infty} e^{i h u} d \mu_{n}(u) \quad \forall h \in R
$$

(Theorem 4.1). We will construct an $f \in \mathfrak{M}^{2}$ in the following manner: define a new sequence of functions by

$$
\begin{gathered}
l_{1}=f_{1}, \quad l_{2}=f_{1}, \quad l_{3}=f_{2}, \\
l_{4}=f_{1}, \quad l_{5}=f_{2}, \quad l_{6}=f_{3}, \text { etc. }
\end{gathered}
$$

For each $l_{n}$, we will let $c_{n}$ be the corresponding covariance function and let

$$
c_{n}^{T}(h)=\frac{1}{2 T} \int_{-T}^{T} l_{n}(x+h) \overline{l_{n}(x)} d x .
$$

Let $A_{1}=0$. Suppose we have chosen $A_{n}$ and have defined $f$ on $\left[0, A_{n}\right]$; let $B_{n}>A_{n}$ be such that

$$
\left|\frac{1}{2 B_{n}} \int_{-A_{n}}^{A_{n}} f(x+h) \overline{f(x)} d x\right| \leqslant \frac{1}{2^{n}} \quad \forall h \in\left[-2^{n}, 2^{n}\right] .
$$


Define $f=0$ on $\left[A_{n}, B_{n}\right] \cup\left[-B_{n},-A_{n}\right]$. Since $c_{n}^{T} \rightarrow c_{n}, T \rightarrow \infty$ uniformly on compact sets, there exists $T_{n}>B_{n}$ such that for $T>T_{n}$,

$$
\left|\frac{T-B_{n}}{T} c^{T-B_{n}}(h)-c_{n}(h)\right| \leqslant \frac{1}{2^{n}} \quad \forall h \in\left[-2^{n}, 2^{n}\right] .
$$

Let $A_{n+1}=T_{n}^{2}$ and let

$$
f(x)= \begin{cases}l_{n}\left(x-B_{n}\right), & \text { for } x \in\left[B_{n}, A_{n+1}\right], \\ l_{n}\left(x+B_{n}\right), & \text { for } x \in\left[-A_{n+1},-B_{n}\right] .\end{cases}
$$

Inductively, we can define the function $f$ on $R$. For each fixed $m$, there exists infinitely many $c_{n}$ equal to $c_{m}$. For each fixed compact subset $K$ in $R$, we can show that the conditions in Lemma 4.2 are satisfied (with $c(h)$ replaced by $c_{m}(h)$ ). Hence there exists a sequence $\left\{\varepsilon_{n}\right\}$ which converges to zero and

$$
\lim _{n \rightarrow \infty} \frac{1}{2 \varepsilon_{n}} \int_{-\infty}^{\infty} e^{i h u}\left|g\left(u+\varepsilon_{n}\right)-g\left(u-\varepsilon_{n}\right)\right|^{2} d u=c_{m}(h) \quad \forall h \in K .
$$

By using a diagonal method, we can actually obtain a sequence $\varepsilon_{n}^{\prime}$ such that

$$
\lim _{n \rightarrow \infty} \frac{1}{2 \varepsilon_{n}^{\prime}} \int_{-\infty}^{\infty} e^{i h u}\left|g\left(u+\varepsilon_{n}^{\prime}\right)-g\left(u-\varepsilon_{n}^{\prime}\right)\right|^{2} d u=c_{m}(h) \quad \forall h \in R .
$$

This implies that

$$
\lim _{n \rightarrow \infty} \int_{-\infty}^{\infty} e^{i h u} d \mu_{\varepsilon_{n}^{\prime}}(u)=\int_{-\infty}^{\infty} e^{i h u} d \mu_{m}(u)
$$

Hence $\left\{\mu_{n}\right\} \subseteq D_{g}$.

REMARK 1. If the sequence $\left\{\mu_{n}\right\}$ in the above proposition is supported by a compact set, then $\left\{\mu_{n}\right\}$ is a precompact set in the weak topology induced by $C_{b}(R)$, where $C_{b}(R)$ is the space of bounded continuous functions on $R$. This implies that the corresponding continuous positive definite function $\left\{c_{n}\right\}$ is a precompact subset in the topology of uniform convergence on compact sets $[4, \S 5]$. Hence we can show that $c_{n}(h) \rightarrow c_{n}(0)$ uniformly on $n$ as $h \rightarrow 0$ and $\left\|\tau_{h} f_{n}-f_{n}\right\| \rightarrow 0$ uniformly on $n$ as $h \rightarrow 0$. These and the construction of $f$ imply that $\left\|\tau_{h} f-f\right\| \rightarrow 0$ as $h \rightarrow 0$, i.e. $f \in \mathfrak{N}_{c}^{2}$.

REMARK 2. Each $f_{n}$ in the above proposition is in the class $\mathscr{Q} f^{2}$, hence each $f_{n}$ is in $\mathfrak{M}_{r}^{2}$. It follows from the construction that $f$ is also in $\Re_{r}^{2}$.

5. Multipliers. (A) On $\Re_{c}^{2}$. Let $\mathscr{Q}^{1 / 2}$ be defined as in $\S 2$. For each $\phi \in \mathscr{Q}^{1 / 2}$, we consider $\phi$ as a bounded linear operator on $\mathcal{V}^{2}$ defined by $\phi \cdot g$. Let $\mathcal{V}_{c}^{2}$ denote the image of $\mathfrak{M}_{c}^{2}$ under $W$. Let $\mathcal{T}_{c}$ denote the strong operator topology on $\mathscr{Q}^{1 / 2}$ and on $C_{b}(R)$, the space of bounded continuous functions on $R$ and let $Q \mathcal{C}$ denote the topology of uniform convergence on compact subsets of $R$.

Proposition 5.1. On $\mathscr{D}^{1 / 2}$, the topology $\mathcal{T}_{c}$ is stronger than the topology $\mathscr{Q}$, and the two topologies coincide on bounded subsets of $\mathscr{D}^{1 / 2}$. 
PROOF. Let $D_{k}$ be the set of point mass measures $\delta_{r}$ where $r$ is a rational in $[-k, k]$. Proposition 4.3 and its Remark 1 yields a function $g_{0} \in \mathcal{V}_{c}^{2}$ with $D_{g_{0}} \subseteq D_{k}$. Hence for any $\phi \in \mathscr{Q}^{1 / 2}$,

$$
\begin{aligned}
\left\|\phi \cdot g_{0}\right\|_{\mathscr{V}^{2}}^{2} & =\varlimsup_{\varepsilon \rightarrow 0^{+}} \frac{1}{2 \varepsilon} \int_{-\infty}^{\infty}|\phi(u)|^{2}\left|g_{0}(u+\varepsilon)-g_{0}(u-\varepsilon)\right|^{2} d u \quad \text { (by } \\
& =\varlimsup_{\varepsilon \rightarrow 0^{+}} \int_{-\infty}^{\infty}|\phi(u)|^{2} d \mu_{\varepsilon}(u)=\sup _{\mu \in D_{g_{0}}} \int_{-\infty}^{\infty}|\phi(u)|^{2} d \mu(u) \\
& \geqslant \sup _{\mu \in D_{k}} \int_{-\infty}^{\infty}|\phi(u)|^{2} d \mu(u)=\sup _{\mu \in[-k, k]}|\phi(u)|^{2}
\end{aligned}
$$

This implies that $\sigma_{c}$ is stronger than $\mathscr{U}$. To prove the second assertion, let $B$ be a bounded subset in $\mathscr{D}^{1 / 2}$ and let $\left\{\phi_{n}\right\}, \phi$ be in $B$ with $\phi_{n} \rightarrow \phi$ in $\mathscr{Q}$. Let $g \in \mathscr{V}_{c}^{2}$; then for any $\eta>0$, there exists $A_{0}$ and $\varepsilon_{0}$ such that for $A>A_{0}, 0<\varepsilon<\varepsilon_{0}$,

$$
\frac{1}{2 \varepsilon} \int_{-\infty}^{-A}+\int_{A}^{\infty}|g(u+\varepsilon)-g(u-\varepsilon)|^{2} d u<\frac{\eta}{4 k} \quad \text { (by Theorem 2.3) }
$$

where $k$ is the bound of functions in $B$. Let $n_{0}$ be such that for $n>n_{0}$,

$$
\sup _{x \in[-A, A]}\left|\left(\phi_{n}-\phi\right)(x)\right|<\frac{\eta}{2\|g\|} .
$$

Then for $n>n_{0}$,

$$
\begin{aligned}
&\left\|\left(\phi_{n}-\phi\right) \cdot g\right\|_{\mathcal{V}^{2}}= \varlimsup_{\varepsilon \rightarrow 0^{+}} \frac{1}{2 \varepsilon} \int_{-\infty}^{\infty}\left|\phi_{n}(u)-\phi(u)\right|^{2}|g(u+\varepsilon)-g(u)|^{2} d u \\
& \leqslant \frac{\eta}{2\|g\|} \varlimsup_{\varepsilon \rightarrow 0^{+}} \frac{1}{2 \varepsilon} \int_{-A}^{A}|g(u+\varepsilon)-g(u-\varepsilon)|^{2} d u \\
&+2 k \lim _{\varepsilon \rightarrow 0^{+}} \frac{1}{2 \varepsilon} \int_{-\infty}^{-A}+\int_{A}^{\infty}|g(u+\varepsilon)-g(u-\varepsilon)|^{2} d u \\
&<\eta
\end{aligned}
$$

This completes the proof.

Let $\phi \in C_{b}(R)$; there exists a bounded sequence of functions $\left\{\phi_{n}\right\}$ in $\mathscr{D}^{1 / 2}$ converging to $\phi$ uniformly on compact sets. By Proposition 5.1, $\phi$ defines an operator on $\mathfrak{V}_{c}^{2}$ by $\phi \cdot g=\lim _{n \rightarrow \infty} \phi_{n} \cdot g$ (the limit is taken in the sense of $\mathfrak{V}^{2}$ ). It follows from (2.1) and again Proposition 5.1 that

$$
\|\phi \cdot g\|_{\mathcal{V}^{2}}=\varlimsup_{\varepsilon \rightarrow 0^{+}} \frac{1}{2 \varepsilon} \int_{-\infty}^{\infty}|\phi(u)|^{2}|g(u+\varepsilon)-g(u-\varepsilon)|^{2} d u .
$$

As a consequence, we have

TheOREM 5.2. The space $\mathscr{Q}^{1 / 2}$ in Proposition 5.1 can be replaced by $C_{b}(R)$.

Let $\left\langle\tau_{h}\right\rangle^{-}$denote the strong operator closure of the subspaces generated by the set of translation operators. Let $\left\langle\tau_{h} f\right\rangle^{-}$be the closed subspace generated by $\tau_{h} f$, $h \in R$. An operator $\Phi: \Re_{c}^{2} \rightarrow \Re_{c}^{2}$ is called subordinative $[3,10]$ if $\Phi(f) \in\left\langle\tau_{h} f\right\rangle^{-}$ for all $f \in \Re_{c}^{2}$. For each $\Phi: \Re_{c}^{2} \rightarrow \Re_{c}^{2}$ we will use $\Phi^{\wedge}$ to denote the unique operator on $\widetilde{V}_{c}^{2}$ satisfying $W(\Phi f)=\hat{\Phi(W f)}, f \in \mathfrak{M}_{c}^{2}$. 
THEOREM 5.3. Let $\Phi$ be an operator on $\mathfrak{\Re}_{c}^{2}$; then the following conditions are equivalent:

(i) $\Phi$ is subordinative;

(ii) $\Phi \in\left\langle\tau_{h}\right\rangle^{-}$;

(iii) $\Phi$ belongs to the strong operator closure of the set of convolution operators;

(iv) there exists $a \phi \in C_{b}(R)$ such that $\bar{\Phi} g=\phi \cdot g \forall g \in \mathcal{V}_{c}^{2}$.

We remark that Bertrandias has proved that $\Phi$ is subordinate on $\Re_{r c}^{2}$ if and only if $\Phi$ corresponds to a $\phi \in C_{b}(R)$ through some abstract representations [3].

Proof. (ii) $\Rightarrow$ (i) is clear. To prove (i) $\Rightarrow$ (ii), we suppose $\Phi \notin\left\langle\tau_{h}\right\rangle^{-}$; there exist $f \in \mathfrak{N}_{c}^{2}, l \in\left(\mathfrak{N}_{c}^{2}\right)^{*}$ such that $\langle\Phi f, l\rangle>\sup _{h \in R}\left\langle\tau_{h} f, l\right\rangle$. This implies that $\Phi f$ is not in the closed subspace generated by $\tau_{h} f, h \in R$, and is a contradiction.

(ii) $\Rightarrow$ (iii) is obvious.

(iii) $\Rightarrow$ (iv). Let $M$ denote the set of bounded regular Borel measures on $R$ and let

$$
M_{1}=\left\{\mu \in M: \int|x| d|\mu|<\infty\right\} .
$$

Let $\hat{M}, \hat{M_{1}}$ denote the Fourier-Stieltjes transformation of the measures in $M$ and $M_{1}$ respectively. It is known that $\hat{M_{1}}$ is dense in $\left(\hat{M_{1}}\right)^{-}$under the $\mathscr{Q}$ topology. By Theorem 5.2, $\hat{M_{1}}$ is dense in $\left(\hat{M_{1}}\right)^{-}$under the strong operator topology $\sigma_{c}$. Hence for any $\Phi \in M^{-}$, there exists a sequence $\left\{\mu_{n}\right\}$ in $M_{1}$ such that $\mu_{n} \rightarrow \Phi$ in $\mathcal{T}_{c}$. This implies $\hat{\mu}_{n} \rightarrow \phi$ in $\mathcal{Q} \mathcal{C}$ for some function $\phi \in C_{b}(R)$. By Proposition 2.2 and that $\hat{\mu}_{n} \in \mathscr{Q}^{1 / 2}$, we have

$$
\hat{\Phi g}=\lim _{n \rightarrow \infty} \hat{\mu}_{n}(g)=\lim _{n \rightarrow \infty} \hat{\mu}_{n} \cdot g=\phi \cdot g \quad \forall g \in \mathscr{V}_{c}^{2} .
$$

(iv) $\Rightarrow$ (ii). Note that $\left\langle\tau_{h}{ }^{\wedge}\right\rangle^{-}$contains all trigonometric polynomials, and by Theorem 5.2, $\left\langle\tau_{h}{ }^{\wedge}\right\rangle^{-}=C_{b}(R)$.

We will call an operator $\Phi$ on $\Re_{c}^{2}$ a multiplier if it satisfies either one of the above conditions.

(B) On $\Re_{r c}^{2}$. In the proof of Proposition 5.1, the function $f$ we have constructed can actually be taken in $\Re_{r c}^{2}$ (Remarks 1,2 of Proposition 4.3). Hence all the results in $\S 5(\mathrm{~A})$ can be carried to $\mathfrak{N}_{r c}^{2}$. In the following, we will give one more characterization of the multipliers on $\Re_{r c}^{2}$ concerning the commutation of $\Phi$ and $\tau_{h}, h \in R$.

LEMMA 5.4. Let $f \in \mathfrak{M}_{c}^{2}$,

$$
c_{T}(h)=\frac{1}{2 T} \int_{-T}^{T} f(x+h) \overline{f(x)} d x
$$

and $\mu_{T}$ be the measure defined by $c_{T}(h)=\int_{R} e^{i h u} d \mu(u), h \in R$. Then for any $\varepsilon>0$, there exists an $A$ such that $\mu_{T}(R \backslash[-A, A])<\varepsilon$ for all $T>T_{0}$.

Proof. The proof is similar to Theorem 2.3; we let

$$
p_{n}(x)=\sqrt{\frac{2}{\pi}} \frac{n}{n^{2} x^{2}+1},
$$


then $\left\|p_{n}\right\|_{1}=1$ and $\hat{p_{n}}(u)=e^{-|u| / n}$. By a standard argument, we can show that for any $\varepsilon>0$, there exists $T_{0}, n_{0}$ such that for $T>T_{0}, n>n_{0},\left|c_{T}(0)-p_{n} * c_{T}(0)\right|<\varepsilon$. Since

$$
c_{T}(0)-p_{n} * c_{T}(0)=\int_{R}\left(1-e^{-|u| / n}\right) d \mu_{T}(u)
$$

and $\left|1-e^{-|u| / n}\right| \geqslant \frac{1}{2}$ for $|u|>n$, we conclude that for any $n>n_{0}, T>T_{0}$,

$$
\mu_{T}\left(R \backslash\left[-n_{0}, n_{0}\right]\right)<\varepsilon \quad \forall T>T_{0} .
$$

Lemma 5.5 [7, THeOREM 5.2]. Let $L$ be the set of bounded linear functionals $l$ on $\mathrm{OR}^{2}$ that can be represented as

$$
\langle l, f\rangle=\int_{1}^{\infty}\left(\frac{1}{2 T} \int_{-T}^{T} f(x) \overline{g(x)} d x\right) d \mu(T), \quad f \in \mathfrak{N}^{2},
$$

where $g \in \mathfrak{M}^{2}$, and $\mu$ is a finitely additive regular Borel measure vanishing on bounded subsets of $R$. Then $L$ is norm dense in $\left(\Re^{2}\right)^{*}$.

Let $M^{2}=\left\{f: \sup _{T>1} \frac{1}{2 T} \int_{-T}^{T}|f|^{2}<\infty\right\}$, then $L^{2}(R)$ is contained in $M^{2}$ and the Marcinkiewicz space $\pi^{2}$ can be identified with the quotient space $M^{2} / N$ where $N=\left\{f:\|f\|_{\mathfrak{R}^{2}}=0\right\}$ [7, Proposition 2.4].

THEOREM 5.6. Let $\Phi: M^{2} \rightarrow M^{2}$ be a linear mapping. Suppose $\Phi$ defines a bounded linear operator on $\mathfrak{\pi}_{r c}^{2}$ (we still use the notation $\Phi$ ), and the restriction of $\Phi$ on $L^{2}(R)$ is bounded as an $L^{2}(R)$-operator. Then $\Phi$ is a multiplier on $\Re_{r c}^{2}$ if and only if:

(i) $\Phi \tau_{h}=\tau_{h} \Phi \forall h \in R$;

(ii) $\lim _{T \rightarrow \infty} \frac{1}{2 T} \int_{-\infty}^{\infty}\left|\chi_{T} \Phi(f)-\Phi\left(\chi_{T} f\right)\right|^{2}=0$,

where $\chi_{T}$ is the characteristic function of $[-T, T]$.

Proof. Suppose $\Phi$ is a multiplier; it is clear that (i) is satisfied. To prove (ii), we recall that in [8, Lemma 2.2] we proved that if $\mu \in M_{1}$ (the set of measures $\mu \in M$ with $\left.\int|x| d|\mu|(x)<\infty\right)$, then

$$
\lim _{T \rightarrow \infty} \frac{1}{2 T} \int_{-\infty}^{\infty}\left|\chi_{T} \Phi(f)-\Phi\left(\chi_{T} f\right)\right|^{2}=0 .
$$

Let $\left\{\mu_{n}\right\}$ be a sequence in $M_{1}$ such that $\Phi_{\mu_{n}} \rightarrow \Phi$ in the strong operator topology on $\mathfrak{N}_{r c}^{2}$; hence for each $f$,

$$
\lim _{n \rightarrow \infty} \lim _{T \rightarrow \infty} \frac{1}{2 T} \int_{-T}^{T}\left|\left(\Phi_{\mu_{n}}-\Phi\right) f\right|^{2}=0
$$

We need only show that

$$
\lim _{n \rightarrow \infty} \lim _{T \rightarrow \infty} \frac{1}{2 T} \int_{-\infty}^{\infty}\left|\left(\Phi_{\mu_{n}}-\Phi\right)\left(\chi_{T} f\right)\right|^{2}=0 .
$$

By Theorem 5.2, $\left\{\hat{\mu}_{n}\right\}$ converges to $\hat{\Phi}=\phi$ in the $\mathcal{Q} \mathcal{C}$ topology. Since

$$
\frac{1}{2 T} \int_{-\infty}^{\infty}\left|\left(\Phi_{\mu_{m}}-\Phi_{\mu_{n}}\right)\left(\chi_{T} f\right)\right|^{2}=\int_{-\infty}^{\infty}\left|\hat{\mu}_{n}-\hat{\mu}_{m}\right|^{2} d \mu_{T}
$$


Lemma 5.4 and the $\mathcal{Q} \bigodot$ convergence of $\left\{\Phi_{\mu_{m}}\right\}$ implies that for any $\varepsilon>0$, there exists $T_{0}, m_{0}$ such that for $T>T_{0}, m, n>m_{0}$,

$$
\frac{1}{2 T} \int_{-\infty}^{\infty}\left|\left(\Phi_{\mu_{m}}-\Phi_{\mu_{n}}\right)\left(\chi_{T} f\right)\right|^{2}<\varepsilon
$$

That $\left\{\Phi_{\mu_{n}}\right\}$ also converges to $\Phi$ in the strong operator topology on $L^{2}(R)$ implies

$$
\frac{1}{2 T} \int_{-\infty}^{\infty}\left|\left(\Phi_{\mu_{m}}-\Phi\right)\left(\chi_{T} f\right)\right|^{2}<\varepsilon
$$

and equality (5.2).

To prove the sufficiency, we will first show that

$$
\Phi(s * f)=\Phi(s) * f \quad \forall s \in L_{K}^{2}, f \in \Re_{r c}^{2},
$$

where $L_{K}^{2}$ is the set of $L^{2}$-functions on $R$ with compact supports. Let $A$ be the dense set in $\mathfrak{T}^{2 *}$ as in Lemma 5.5 and let $l \in A$ be represented by

$$
\langle l, f\rangle=\int_{1}^{\infty}\left(\frac{1}{2 T} \int_{-T}^{T} f(x) \overline{g(x)} d x\right) d \mu(T)
$$

where $g \in \mathfrak{T}^{2}$ and $\mu$ is finitely additive, vanishes on bounded subsets of $R$.

$$
\begin{aligned}
& \langle l,(\Phi s) * g\rangle=\int_{1}^{\infty} \frac{1}{2 T} \int_{-T}^{T}\left(\int_{-\infty}^{\infty} \tau_{-y}(\Phi s(x)) f(y) d y\right) \overline{g(x)} d x d \mu(T) \\
& =\int_{1}^{\infty} \frac{1}{2 T} \int_{-\infty}^{\infty}\left(\int_{-\infty}^{\infty} \Phi\left(\chi_{T}(x) \tau_{-y} s(x)\right) \cdot \chi_{T}(x) \overline{g(x)} d x\right) f(y) d y d \mu(T) \\
& =\int_{1}^{\infty} \frac{1}{2 T} \int_{-\infty}^{\infty}\left(\int_{-\infty}^{\infty} \chi_{T}(x) \tau_{-y} s(x) \cdot \Phi^{*}\left(\chi_{T}(x) \overline{g(x)}\right) d x\right) f(y) d y d \mu(T) \\
& \quad=\int_{1}^{\infty} \frac{1}{2 T} \int_{-\infty}^{\infty} \chi_{T}(x)(s * f)(x) \cdot \Phi^{*}\left(\chi_{T}(x) \bar{g} \text { is the adjoint operator of } \Phi \text { on } L^{2}(R)\right) \\
& =\int_{1}^{\infty} \frac{1}{2 T} \int_{-\infty}^{\infty} \Phi\left(\chi_{T} \cdot s * f\right)(x) \cdot \chi_{T}(x) \overline{g(x)} d x d \mu(T) \\
& =\int_{1}^{\infty} \frac{1}{2 T} \int_{-T}^{T} \Phi(s * f)(x) \overline{g(x)} d x d \mu(T) \quad(\text { by (i) and the property of } \mu) \\
& =\langle l, \Phi(s * f)\rangle .
\end{aligned}
$$

This implies that $(\Phi s) * f=\Phi(s * f) \forall s \in L_{K}^{2}, f \in \mathfrak{N}_{r c}^{2}$ and $\Phi(s)$ is a convolution operator on $\mathfrak{M}_{r c}^{2}$. Theorem 5.3(i) implies that

$$
\Phi(s * f)=\Phi(s) * f \in\left\langle\tau_{h} f\right\rangle^{-} .
$$

By taking $\left\{s_{n}\right\}$ to be a sequence with compact support and which converges to $\delta_{0}$ weakly, we have $\left\|s_{n} * f-f\right\|_{\mathfrak{R}^{2}} \rightarrow 0$ as $n \rightarrow \infty$ (see the proof of Theorem 2.3). This implies that $\Phi(f) \in\left\langle\tau_{h} f\right\rangle^{-}$and hence $\Phi$ is subordinative, i.e. it is a multiplier.

We conclude this subsection by a theorem concerning the norm of the multipliers. 
THEOREM 5.7. Let $\Phi$ be a multiplier on $\mathfrak{T}_{r c}^{2}$ and let $\hat{\Phi}=\phi \in C_{b}(R)$. Then

$$
\|\Phi\|_{\Re_{r c}^{2}}=\|\Phi\|_{\Upsilon_{r c}^{2}}=\|\phi\|_{\infty} \text {. }
$$

Proof. In [7, Theorem 2.4], we proved that if $\Phi$ is a convolution operator on $\mathfrak{N}_{r}^{2}$, i.e. $\Phi=\Phi_{\mu}$ for some $\mu \in M$, then

$$
\left\|\Phi_{\mu}\right\|_{\mathscr{R}_{r}^{2}}=\left\|\Phi_{\mu}\right\|_{L^{2}(R)}=\|\hat{\mu}\|_{\infty} .
$$

The same proof holds for $\Phi_{\mu}$ on $\Re_{r c}^{2}$ (we need only observe that the function $\tilde{f}$ constructed in [7, Lemma 2.3] is in fact in $\Re_{r c}^{2}$ ). Hence $\left\|\Phi_{\mu}\right\|_{\Re_{r c}^{2}}=\|\hat{\mu}\|_{\infty}$. Let $\phi \in C_{b}(R)$; there exists a bounded sequence $\hat{\mu}_{n}$ such that $\hat{\mu}_{n} \rightarrow \phi$ uniformly on compact subsets of $R$ and $\lim _{n \rightarrow \infty}\left\|\hat{\mu}_{n}\right\|_{\infty}=\|\phi\|_{\infty}$. By the lower semicontinuity of the norm with respect to the strong operator topology, we have $\|\Phi\|_{\mathfrak{R}_{r c}^{2}} \leqslant\|\phi\|_{\infty}$. On the other hand, for any $\varepsilon>0$, there exists $u_{0}$ such that $\left|\phi\left(u_{0}\right)\right| \geqslant\|\phi\|_{\infty}-\varepsilon$. Let $f_{0}=e^{i u_{0}}(\cdot)$ and $g_{0}=W\left(f_{0}\right)$, then $D_{g_{0}}=\left\{\delta_{u_{0}}\right\}$ and

$$
\begin{aligned}
\|\Phi\|_{\pi_{r c}^{2}} & \geqslant\left\|\Phi f_{0}\right\|=\left\|\phi \cdot g_{0}\right\| \\
& =\lim _{\varepsilon \rightarrow 0^{+}}\left(\frac{1}{2 \varepsilon} \int_{-\infty}^{\infty}|\phi(u)|^{2}|g(u+\varepsilon)-g(u-\varepsilon)|^{2} d u\right)^{1 / 2} \\
& =\left(\int_{-\infty}^{\infty}|\phi(u)|^{2} d \delta_{u_{0}}(u)\right)^{1 / 2} \geqslant\|\phi\|_{\infty}-\varepsilon .
\end{aligned}
$$

Hence $\left\|\Phi_{\mu}\right\|_{\mathscr{N}_{r c}^{2}}=\|\hat{\mu}\|_{\infty}$. For the norm of $\hat{\Phi}$ on $\mathcal{V}_{r c}^{2}$, we observe that

$$
\begin{aligned}
\|\hat{\Phi g}\|_{{\widetilde{V_{r c}^{2}}}^{2}} & =\lim _{\varepsilon \rightarrow 0^{+}}\left(\frac{1}{2 \varepsilon} \int_{-\infty}^{\infty}|\phi(u)|^{2}|g(u+\varepsilon)-g(u-\varepsilon)|^{2} d u\right)^{1 / 2} \\
& \leqslant\|\phi\|_{\infty} \cdot\|g\|_{\mathscr{\Upsilon}^{2}},
\end{aligned}
$$

i.e. $\|\hat{\Phi}\|_{\mho_{r c}^{2}} \leqslant\|\phi\|_{\infty}$. By using the same argument as above, we have $\|\hat{\Phi}\| \geqslant\|\phi\|_{\infty}$ also. This completes the proof.

(C) On $\Re_{r}^{2}$. In [8, Theorem 2.6], we showed that the strong operator sequential convergence and the norm convergence coincide on the space of convolution operators. That is just a corollary of the following more complete theorem.

THEOREM 5.8. Consider $\mathscr{D}^{1 / 2}$ a class of operators on $\mathfrak{V}_{r}^{2}$ defined by multiplication; then the strong operator topology and the topology of uniform convergence coincide.

Proof. It is clear from (2.1) that the topology of uniform convergence on $\mathscr{D}^{1 / 2}$ is stronger then the strong operator topology on $\mathcal{V}_{r}^{2}$. On the other hand let $D$ be the set of point mass measures $\delta_{r}$ where $r$ is any rational in $R$. Proposition 4.3 implies that there exists an $f \in \mathfrak{M}_{r}^{2}\left(f\right.$ will not be in $\left.\mathfrak{N}_{c}^{2}\right)$ with $D_{g} \supseteq D$, where $g=W(f)$. Hence it follows from the same proof as Proposition 5.1 that

$$
\|\phi \cdot g\|_{\mathfrak{V}^{2}}^{2} \geqslant \sup _{u \in R}|\phi(u)|^{2}
$$

This implies that the strong operator topology on $\mathfrak{V}_{r}^{2}$ is stronger than the topology of uniform convergence. 
By Proposition 2.1 and Theorem 5.8 we have

COROLlARY 5.9. On the space of convolution operators on $\mathfrak{M}_{r}^{2}$ the strong operator topology and the norm topology coincide.

Note that the norm closure of the set $\{\hat{\mu}: \mu \in M\}$ is a proper subset in $C_{b}(R)$. Hence the equivalent conditions in Theorem 5.3 will not hold on $\mathfrak{\pi}_{r}^{2}$.

\section{A Tauberian theorem.}

LEMma 6.1. Let $\phi$ be a multiplier on $\Re_{c}^{2}$ such that $\hat{\Phi}=\mu$ is nonvanishing on $R$. Let $f \in \mathfrak{N}_{c}^{2}$ such that $\|\Phi(f)\|=0$. Then $g=W(f)$ satisfies

$$
\lim _{\varepsilon \rightarrow 0^{+}} \frac{1}{\varepsilon} \int_{-A}^{A}|g(u+\varepsilon)-g(u-\varepsilon)|^{2} d u=0 \quad \forall A>0 .
$$

Proof. Since $\Phi$ is a multiplier, $\phi$ is continuous. For any $A>0$ there exists a $Q>0$ such that $|\hat{\phi}(u)|>Q$ for all $u \in[-A, A]$. Hence

$$
\begin{aligned}
& \varlimsup_{\varepsilon \rightarrow 0} \frac{Q^{2}}{2 \varepsilon} \int_{-A}^{A}|g(u+\varepsilon)-g(u-\varepsilon)|^{2} d u \\
& \quad \leqslant \lim _{\varepsilon \rightarrow 0} \frac{1}{\varepsilon} \int_{-\infty}^{\infty}|\hat{\phi}(u)|^{2} \cdot|g(u+\varepsilon)-g(u-\varepsilon)|^{2} d u \\
& \quad=\|W(\mu * f)\|_{\mathscr{V}^{2}}^{2} \leqslant\|W\|^{2} \cdot\|\mu * f\|_{\pi^{2}}^{2}=0 . \quad \square
\end{aligned}
$$

The following theorem generalizes a result of Wiener [16, Theorem 29 and 8, Theorem 4.3].

THEOREM 6.2. Let $\Phi$ be a multiplier on $\Re_{c}^{2}$ such that $\Phi \hat{\Phi}=\phi$ is nonvanishing. Let $f \in \Re_{c}^{2}$ satisfy

$$
\lim _{T \rightarrow \infty} \frac{1}{2 T} \int_{-T}^{T}|\Phi f|^{2}=0
$$

Then for any multiplier $\Psi$ on $\Re_{c}^{2}$,

$$
\lim _{T \rightarrow \infty} \frac{1}{2 T} \int_{-T}^{T}|\Psi f|^{2}=0
$$

Proof. Let $\psi=\hat{\Psi}$ and let $g=W(f)$. By Lemma 6.1, we have for any $A>0$

$$
\begin{aligned}
\lim _{\varepsilon \rightarrow 0^{+}} \frac{1}{2 \varepsilon} \int_{-A}^{A}|\psi(u)|^{2} \cdot|g(u+\varepsilon)-g(u-\varepsilon)|^{2} d u \\
\quad \leqslant \sup _{|u|<A}|\psi(u)|^{2} \cdot \lim _{\varepsilon \rightarrow 0^{+}} \frac{1}{2 \varepsilon} \int_{-A}^{A}|g(u+\varepsilon)-g(u-\varepsilon)|^{2} d u=0
\end{aligned}
$$

and

$$
\begin{aligned}
\lim _{A \rightarrow \infty} & \varlimsup_{\varepsilon \rightarrow 0^{+}} \frac{1}{2 \varepsilon} \int_{-\infty}^{-A}+\int_{A}^{\infty}|\psi(u)|^{2} \cdot|g(u+\varepsilon)-g(u-\varepsilon)|^{2} d u \\
& \leqslant \sup _{|u|>A}|\psi(u)|^{2} \cdot \varlimsup_{\varepsilon \rightarrow 0^{+}} \frac{1}{2 \varepsilon} \int_{-\infty}^{-A}+\int_{A}^{\infty}|g(u+\varepsilon)-g(u-\varepsilon)|^{2} d u=0 .
\end{aligned}
$$


The two estimations imply that $\|\psi g\|_{\mho^{2}}=0$, i.e.

$$
\lim _{T \rightarrow \infty} \frac{1}{2 T} \int_{-T}^{T}|\Phi f|^{2}=0
$$

\section{REFERENCES}

1. K. Anzai, S. Koizumi and K. Matsuoka, On the Wiener formula of functions of two variables, Tokyo J. Math. (to appear).

2. J. Bertrandias, Espaces de fonctions bornées et continues en moyenne asymptotique d'ordre p, Bull. Soc. Math. France 5 (1966).

3. __ Opérateurs subordinatifs sur des espaces de fonctions bornées en moyenne quadratique, J

Math. Pures Appl. 52 (1973), 27-63.

4. N. Bourbaki, Intégration, Chapitre 9, Hermann, Paris, 1969.

5. G. Hardy and J. Littlewood, Some properties of fractional integrals, Math. Z. 27 (1928), 565-606.

6. R. Larsen, An introduction to the theory of multipliers, Springer-Verlag, Berlin and New York, 1971.

7. K. Lau, On the Banach spaces of functions with bounded upper means, Pacific J. Math. 91 (1980), $153-173$.

8.

, The class of convolution operators on the Marcinkiewicz spaces, Ann. Inst. Fourier (Grenoble) 31 (1981), 225-243.

9. K. Lau and J. Lee, On generalized harmonic analysis, Trans. Amer. Math. Soc. 259 (1980), 75-97.

10. J. Marcinkiewicz, Une remarque sur les espaces de M. Besicovitch, C. R. Acad. Sci. Paris Ser. A-B 208 (1939), 157-159.

11. P. Masani, The normality of time invariant, subordinative operators in a Hilbert space, Bull. Amer. Math. Soc. 71 (1965), 546-550.

12.

, Commentary on the memoire on generalized harmonic analysis [30a], Norbert Wiener:

Collected Work, M.I.T. Press, Cambridge, Mass., 1979, pp. 333-379.

13. N. Wiener, Generalized harmonic analysis, Acta Math. 55 (1930), 117-258.

14. Tauberian theorems, Ann. of Math. (2) 33 (1932), 1-100.

15. Extrapolation, interpolations and smoothing of stationary time series, M.I.T. Press, Cambridge, Mass. and Wiley, New York, 1949.

16. _ The Fourier integral and certain of its applications, Dover, New York, 1959.

Department of Mathematics and Statistics, University of Pittsburgh, Pittsburgh, PennsylVANIA 15260 